\title{
The effect of segregation of flowering time on fine-scale spatial genetic structure in an alpine-snowbed herb Primula cuneifolia
}

\author{
AS Hirao and G Kudo \\ Graduate School of Environmental Earth Science, Hokkaido University, Sapporo, Japan
}

\begin{abstract}
The flowering phenology of alpine-snowbed plants varies widely depending on the time of snowmelt. This variation may cause spatial and temporal heterogeneity in pollen dispersal, which in turn may influence genetic structure. We used spatial autocorrelation analyses to evaluate relative effect of segregation in flowering time and physical distance on fine-scale spatial genetic structure (SGS) of a snowbed herb Primula cuneifolia sampled in 10-m grids within a continuous snow patch $(110 \times 250 \mathrm{~m})$ using nine allozyme loci. Although the individual flower lasts for $\leqslant 10$ days, flowering season varied over 50 days from late June to the middle of August within the plot. The effect of flowering phenology on
\end{abstract}

SGS was assessed using spatial autocorrelation analyses based on the pairwise kinship coefficients for all sampled plants (control pairs), plants with flowering overlap (co-flowering pairs) and plants with separate flowering season (non-coflowering pairs). The degree of SGS increased as the extent of flowering segregation increased: co-flowering pairs $<$ control pairs $<$ non-co-flowering pairs, indicating substantial effect of restriction in gene flow due to phenological heterogeneity. Flowering segregation caused by snowmelt timing is a critical factor for reinforcing the fine-scale SGS in this species. Heredity (2008) 100, 424-430; doi:10.1038/hdy.2008.1; published online 13 February 2008

Keywords: flowering phenology; gene flow; pollen dispersal; snowmelt gradient; spatial genetic structure

\section{Introduction}

A restriction of gene flow is a key determinant in the establishment of fine-scale spatial genetic structures (SGS) in plant populations (Vekemans and Hardy, 2004). Isolation by distance, that is, decreasing genetic relatedness among pairwise individuals with increasing physical distance (Wright, 1943), is a driving force that shapes local kinship structure, which in turn increases the likelihood of mating events between related individuals (Grifin and Eckert, 2003; Degen et al., 2004; Herlihy and Eckert, 2004). Some empirical studies have detected significant SGS when seed dispersal and/or pollen dispersal is restricted (for example, Hamrick et al., 1993; Epperson and Alvarez-Buylla, 1997; Hardy et al., 2006). In a simulation study, SGS was predicted to develop solely via a sufficient restriction of gene flow, without natural selection or other determinant forces (Turner et al., 1982). In addition, Ohsawa et al. (1993) simulated different degrees of limitation to pollen flow and the consequent genetic structure. They found that genetic structure was very sensitive to the degree of pollen flow and that the spatial autocorrelation coefficient increased as pollen flow was limited, especially around neighborhoods (Ohsawa et al., 1993). In natural populations, however, few studies have assessed

Correspondence: Dr AS Hirao, Graduate School of Environmental Earth Science, Hokkaido University, Sapporo 060-0810, Japan.

E-mail: hirao@ees.hokudai.ac.jp

Received 28 May 2007; revised 11 October 2007; accepted

7 December 2007; published online 13 February 2008 to what extent a limitation of gene flow reinforces the fine-scale SGS.

Variation in the timing of flowering within a local site often influences the pattern of pollen-mediated gene flow and in turn the SGS (Kitamoto et al., 2006). Alpine-snowbed plants are suitable for examining this aspect. The date of flowering of snowbed plants is determined by the time of snowmelt so that heterogeneous flowering patterns occur along a snowmelt gradient within a local site (Holway and Ward, 1965; Kudo, 1991, 1996). Flowering phenology of a single species often varies $>1$ month along a steep snowmelt gradient. Thus, segregation in flowering time (phenological isolation) among individual plants and/or populations can act as a barrier to pollen dispersal. Such temporal heterogeneity in pollen dispersal, in addition to the simple isolation by distance, enabled us to reveal the impact of pollen-mediated gene flow on the fine-scale SGS of a plant population.

In a previous study, Stanton et al. (1997) analyzed the microgeographic genetic structure of an alpine-snowbed herb, Ranunculus adoneus, along a snowmelt gradient. Although their evidence of genetic structure along a snowmelt gradient was not clear, their statistical approach was based on bivariate regression limits rather than a spatial autocorrelation analysis as used in other studies (reviewed in Heywood, 1991; Epperson, 1993). Recently, fine-scale SGS has been quantified with the $S p$ statistic based on spatial autocorrelation analysis (Dutech et al., 2005; Hardy et al., 2006; reviewed in Vekemans and Hardy, 2004). The Sp statistic, which is primarily a function of a decreasing rate of genetic relatedness 
among pairwise individuals with logarithmic distance, can be expressed in term of Wright's neighborhood size (Vekemans and Hardy, 2004). Thus, the Sp statistic can readily be used to compare the degree of SGS across species and studies. We used the $S p$ statistic to evaluate relative effect of segregation in flowering time and physical distance on fine-scale SGS of an alpine-snowbed herb, Primula cuneifolia Ledeb. (Primulaceae). P. cuneifolia has the following advantages. First, its rapid flowering after snowmelt and short flower longevity create a clear phenological sequence along a snowmelt gradient (Kudo, 1991, 1992; Kudo and Hirao, 2005). Second, because of the heterostylous mating system like other Primula species (Richards, 2002), the effect of selfing on SGS is negligible.

In this study, we investigated the SGS among $P$. cuneifolia individuals separated by $10-\mathrm{m}$ grids in a continuous snow patch $(110 \times 250 \mathrm{~m})$. Because the snowmelt sequence is consistent across years, pollen-mediated gene flow along a snowmelt gradient may be stable enough to shape genetic structure. We hypothesize that the degree of SGS partitioned by pairwise plants with separate flowering season (non-co-flowering pairs) was greater than that by pairs with flowering overlap (co-flowering pairs) because limitation of gene flow could reinforce SGS. Heterogeneity in the timing of pollen dispersal may be critical factor to shape SGS. Spatial autocorrelation analyses incorporating flowering time can be used to determine the significance of pollen-mediated gene flow. Our objective was to examine how the extent of segregation in flowering time affects fine-scale SGS.

\section{Materials and methods}

\section{Plant species}

$P$. cuneifolia is a diploid perennial herb $(2 n=22)$ distributed in the north Pacific region. Each ramet typically has 1-4 flower buds, which were formed the previous season. Pre-flowering period is substantially determined by the cumulative temperature above $5{ }^{\circ} \mathrm{C}$ (Kudo, 1991), and flowering usually starts about 10 days after snowmelt (Kudo, 1992) when snow disappears after late June. An individual flower lasts for $\leqslant 10$ days. Major pollinators are bumblebees (Bombus spp.), but lepidopteran insects (for example, Aporia crataegi and Aglais urticae) occasionally visit. This species has heterostylous flowers of two floral morphs, pin and thrum, and it has intramorph incompatibility (AS Hirao, unpublished data). Each capsule contains several dozen seeds, which are dispersed by gravity around the maternal plants. This species occasionally grows clonally as dividing axillary buds. However, clonal patches larger than $1 \mathrm{~m}$ were not detected by a visual census of genet-specific pattern of floral guide mark (AS Hirao, personal observation). Thus, the clonality did not affect following analysis.

\section{Study system and flowering phenology}

A survey plot was established in 2003 in a continuous snow patch on a gentle slope at Kaun-daira, the central part of the Taisetsu Mountains in Hokkaido, northern Japan (for details, see Hirao and Kudo, 2004). After snowmelt, more than 10000 flowering individuals of $P$. cuneifolia occurred within the plot $(110 \times 250 \mathrm{~m})$, where flowering density was about 1-5 inflorescences per $\mathrm{m}^{2}$. We divided the large snowbed into $10-\mathrm{m}$ grids. Flowering within each grid point was recorded at 3-10 days intervals (Figure 1). The peak flowering time, an index of flowering phenology, was calculated as the median date between the first and end recognition of flowering within the individual grid cells. For genetic analysis, leaf material was collected from one flowering individual nearest to the center of each grid, unless no flowering individual was located within $2 \mathrm{~m}$ area of the grid point $(N=140)$. To clarify how extent flowering segregation depends on physical distance in the plot, flowering time autocorrelations against physical distance of pairwise individuals were calculated using Moran's I-statistic (Moran, 1950). The physical distance classes were set
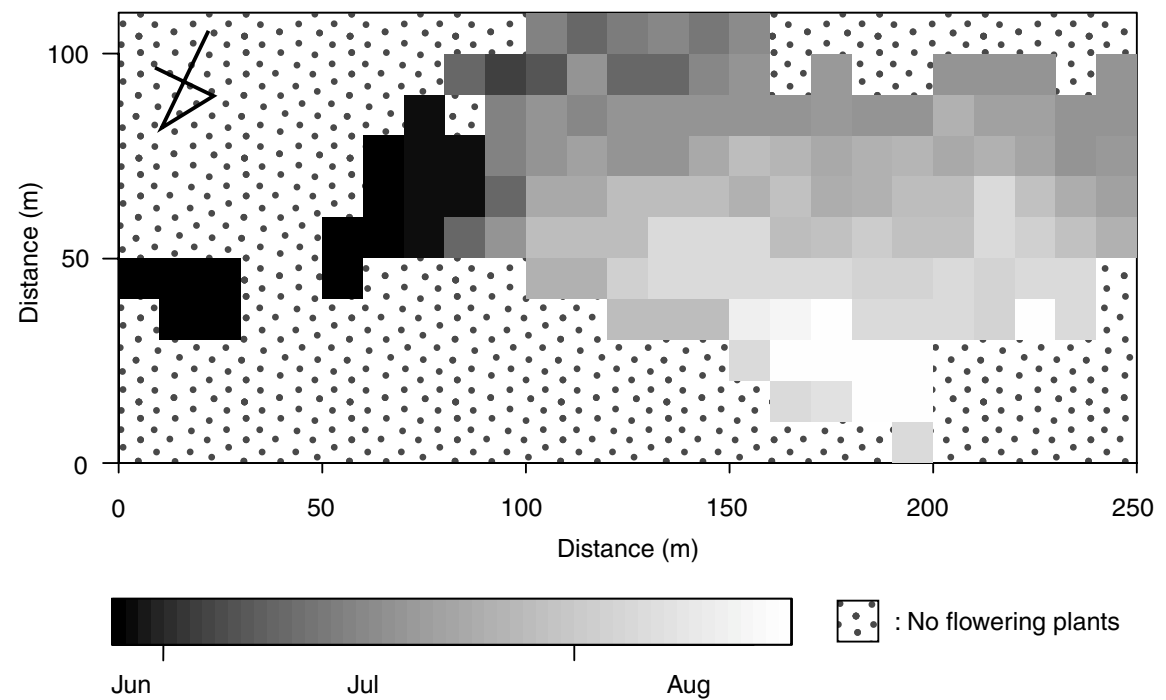

Figure 1 Flowering phenology for plants of Primula cuneifolia within a continuous snow patch. Flowering time is represented by a continuum shading from black (late June at early snowmelt) to white (mid August at late snowmelt). For allozyme analysis, one flowering individual was sampled at the center of each $10 \times 10 \mathrm{~m}$ grid $(N=140)$. 
by the upper limit of the distance class as follows: 10, 15, $20,30,40,50,60,70,80,90,100$ and $250 \mathrm{~m}$.

\section{Allozyme protocols}

Leaf samples were placed on ice and stored at $-78^{\circ} \mathrm{C}$ prior to electrophoresis. Approximately $50 \mathrm{mg}$ of leaf tissue was homogenized in $2.0 \mathrm{ml}$ extraction buffer $(0.1 \mathrm{M}$ Tris- $\mathrm{HCl}(\mathrm{pH} 7.5), 20 \%(\mathrm{v} / \mathrm{v})$ glycerol, $0.75 \%$ Tween 80 , $10 \mathrm{mM}$ dithiothreitol, $0.1 \%$ (v/v) $\beta$-mercaptoethanol, $0.2 \%(\mathrm{w} / \mathrm{v})$ bovine serum albumin and $75 \mathrm{mg} \mathrm{ml}^{-1}$ polyvinylpolyprrolidone). The extracts were loaded on polyacrylamide vertical slab gels after clarifying by centrifugation at $10000 \mathrm{~g}$ for $30 \mathrm{~min}$ twice. Electrophoresis was conducted at $4{ }^{\circ} \mathrm{C}, 12 \mathrm{~mA} \mathrm{~cm}^{-2}$ for $150 \mathrm{~min}$. Enzyme systems followed published protocols (Shiraishi, 1988; see Tsumura, 2001 for details): alanine aminopeptidase (3.4.11.1; 1 locus), aspartate aminotransferase (2.6.1.1; 2 loci), esterase (3.1.1; 1 locus), fumarase (4.2.1.2; 1 locus), isocitric acid (1.1.1.42; 1 locus), 6-phosphogluconate dehydrogenase (1.1.1.44; 3 loci). We resolved these putative loci under the assumption that they show Mendelian inheritance.

\section{Genetic analysis}

As background for spatial autocorrelation analysis, we estimated the number of alleles per locus $(A)$, the effective number of alleles per locus $\left(A_{\mathrm{e}}\right)$, gene diversity $\left(H_{\mathrm{e}}\right)$ and Wright's inbreeding coefficient $(F$, following Weir and Cokerham, 1984) for the allozyme loci. The significance of $F$ across loci was obtained by randomized procedures using the program FSTAT (Goudet, 1995).

To investigate fine-scale SGS, we conducted spatial autocorrelation analysis based on the kinship coefficient between individuals $i$ and $j$ ( $F_{i j}$, following Loiselle et al., 1995) against physical distance on a logarithmic scale. The kinship coefficient is a measure of the inbreeding coefficient between related individuals, that is, 0.25 between full sibs and 0.125 between half sibs. The physical distance classes were set as same as the estimation of the flowering time autocorrelation. We tested the significance of SGS by comparing the observed slope of the linear regression of the kinship coefficient on the logarithmic-physical distance class, $b$, with those obtained after 9999 permutations of the spatial coordinates for the individuals. In the same way, the confidence interval (95\%) for the average kinship coefficient in a particular distance was obtained from 9999 permutation procedures. The effects of flowering time on SGS were assessed by comparing three types of correlograms: (1) comparing all pairwise individuals (control pairs), (2) comparing those whose peak flowering-time overlapped within 10 days (co-flowering pairs) and (3) those separated by $>10$ days (non-co-flowering pairs). The criterion of phenological category is based on flowering duration of individual plants as mentioned above. These spatial autocorrelation analyses are simplified analogues of the anisotropic autocorrelation analysis for a windpollinated tree Quercus lobata (Dutech et al., 2005), in which physical distance between the individuals was weighted by prevailing wind direction. The overall effect of phenological segregation with that of spatial distance on kinship coefficient was assessed by a partial Mantel test based on Kendall's coefficients, in which the phenological distance was 0 or 1 ( 0 , co-flowering pair;
1, non-co-flowering pair), and the spatial distance was $\log$ transformed.

The degree of SGS was quantitatively evaluated using the statistic $S p=-b /(1-F)$, where $b$ is the regression slope described above, and $F$ is the kinship coefficient for the first distance class (Vekemans and Hardy, 2004). The reciprocal of the $S p$ statistic is an estimate of Wright's neighborhood size (Vekemans and Hardy, 2004). The standard errors for the $S p$ statistics were estimated using the jackknife procedure over loci. In this study, the $S p$ values were estimated for three types of correlograms (control pairs, co-flowering pairs, non-co-flowering pairs). We expected that the degree of SGS partitioned by non-co-flowering pairs was greater than that by coflowering pairs because restriction of gene flow due to flowering segregation could reinforce SGS. To test this prediction, we compared the observed $S p$ value difference among non-co-flowering pairs and co-flowering pairs with a null distribution obtained by permutations of the phenological coordinates of individuals by keeping spatial coordinates and genotype. The null distribution was obtained by 9999 randomization procedures. These analyses were conducted using programs written by AS Hirao in $R$ language ( $R$ Development Core Team, 2006).

\section{Results}

\section{Flowering phenology}

Within the continuous snow patch, snowmelt progressed from the west side, where flowering occurred in late June (Figure 1). Sequential flowering continued toward a microgeographic depression place in the southeast until the middle of August. Thus, the flowering phenology varied across more than 50 days along a snowmelt gradient. The autocorrelation of flowering time among individuals decreased with physical distance from 0.81 in the first distance class to -0.57 in the furthest class (Figure 2a), reflecting directional snowmelt pattern within the plot. Distribution of pairwise physical distance among non-co-flowering pairs highly deviated toward long distance classes in comparison with that among co-flowering pairs (Figure $2 b$ ).

\section{Genetic variation}

Nine loci yielded 24 alleles with the effective number of 1.3. alleles per locus (Table 1). Expected heterozygosity $\left(H_{\mathrm{e}}\right)$ ranged from 0.007 to 0.543 with the average of 0.162 across loci. The mean inbreeding coefficient $(F)$ across loci was significantly positive from the Hardy-Weinberg expectation $(F=0.063 \pm 0.027$ s.e.; $P<0.05$ by randomization test).

\section{Spatial genetic structure}

Of the 9370 pairwise combinations among 140 individuals $(9370=140 \times(140-1) / 2), 4280$ pairs were coflowering, and the other 5450 pairs were non-co-flowering. The correlogram of all pairwise individuals (control pairs) showed that kinship coefficients decreased convincingly with logarithmic physical distance (Figure 3a), where significant SGS occurred $(b=-0.016 ; P<0.001$; Table 2). The SGS partitioned by co-flowering pairs or non-co-flowering pairs also showed significant $(b=-0.012 ; \quad P<0.05$ and $=b=-0.047 ; \quad P<0.001$ for 
a Flowering time autocorrelation against physical distance

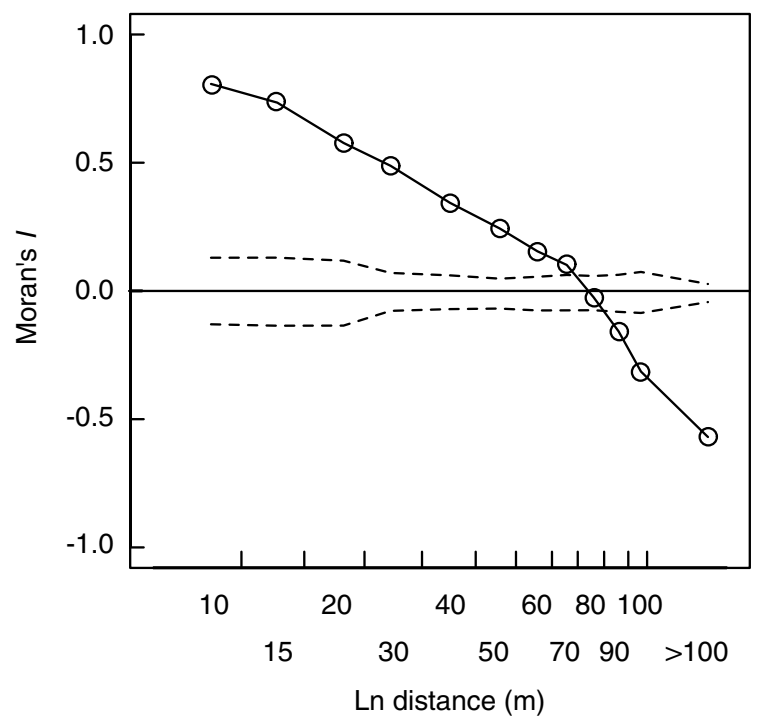

b

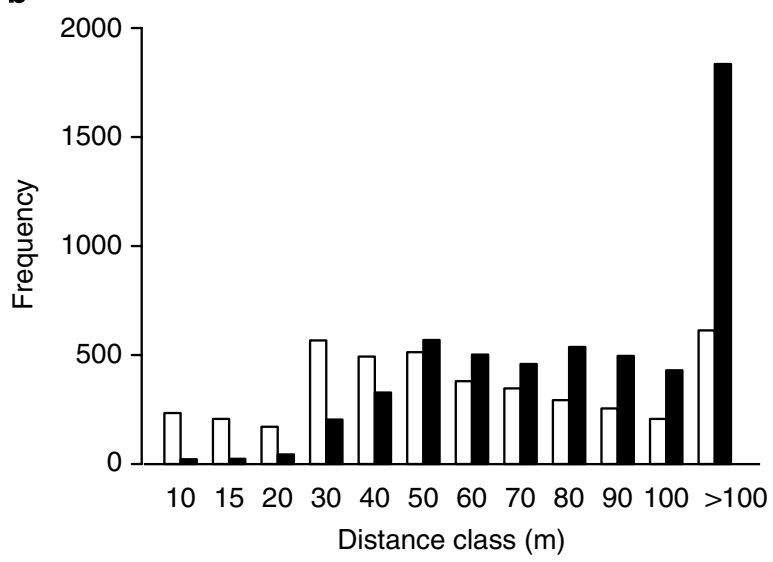

Figure 2 Relationship between heterogeneity in flowering time and physical distance. (a) Correlogram of flowering time autocorrelation against pairwise physical distance on a logarithmic scale (upper limit of the distance class: 10, 15, 20, 30, 40, 50, 60, 70, 80, 90, 100 and $>100 \mathrm{~m}$ ), respectively. Dashed lines represent a $95 \%$ null hypothesis confidence region assuming no autocorrelation of flowering time based on 1000 randomizations (b) Distribution of pairwise physical distance for co-flowering pairs (open bars) and non-co-flowering pairs (closed bars)

co-flowering pairs and non-co-flowering pairs, respectively; Table 2) but showed a contrasting pattern. The kinship coefficients among co-flowering pairs were low, and the genetic relatedness in the first distance class was marginally positive $(F=0.036 \pm 0.009 ; \quad P<0.06$; Figure $3 \mathrm{~b}$ ). In contrast, the kinship coefficients among non-co-flowering pairs were higher in the adjacent neighborhoods (Figure 3c). Especially in the first distance class, the highest kinship coefficient occurred significantly $(F=0.142 \pm 0.019 ; P<0.001)$, regardless of larger range of confidence interval due to small number of non-co-flowering pairs in the class (Figure $2 b$ ).

The $S p$ statistics, the index of the degree of SGS, summarized the difference in the three types of correlograms (control pairs, co-flowering pairs and non-co-flowering pairs; Table 2). The $S p$ values increased
Table 1 Characteristics for nine allozyme loci in Primula cuneifolia $(N=140)$

\begin{tabular}{llccr}
\hline Locus & $A$ & $A_{\mathrm{e}}$ & $H_{\mathrm{e}}$ & \multicolumn{1}{c}{$F$} \\
\hline AAT-1 & 2 & 1.01 & 0.007 & 0.000 \\
AAT-2 & 2 & 1.02 & 0.021 & -0.007 \\
AAP & 3 & 1.78 & 0.445 & 0.082 \\
IDH-2 & 3 & 1.44 & 0.309 & 0.053 \\
6PGD-1 & 4 & 1.03 & 0.028 & 0.246 \\
6PGD-2 & 2 & 1.01 & 0.007 & 0.000 \\
6PGD-3 & 2 & 1.01 & 0.007 & 0.000 \\
FM & 2 & 1.10 & 0.089 & 0.116 \\
EST-1 & 4 & 2.29 & 0.543 & 0.040 \\
Mean & 2.67 & 1.30 & 0.162 & 0.063 \\
s.e. & 0.29 & 0.15 & 0.071 & 0.027 \\
\hline$A, n$ & & &
\end{tabular}

$A$, number of alleles; $A_{\mathrm{e}}$, the effective number of alleles, $H_{\mathrm{e}}$, expected heterozygosity or gene diversity; $F$, Wright's inbreeding coefficient. The mean $F$ across loci was significantly positive according to a randomization test $(P<0.05)$.

with increasing heterogeneity in flowering phenology: co-flowering pairs $<$ control pairs $<$ non-co-flowering pairs. The kinship coefficients for the first distance class, $F$, contributed to this tendency (Table 2). The partial Mantel test showed that the partial regression coefficient for phenological segregation on kinship coefficient was not significant $\left(r_{\text {pheno }}=-0.016 ; P>0.10\right)$, whereas the coefficient for spatial distance was significantly negative $\left(r_{\text {spatial }}=-0.041 ; P<0.05\right)$. However, the permutation test for the $S p$ values detected that the $S p$ value among non-co-flowering pairs was significantly greater than that among co-flowering pairs $(0.055-0.012=0.043$; $P<0.001$ ), indicating substantial effect of restriction in pollen-mediated gene flow. Therefore, flowering segregation caused by snowmelt timing is a critical factor contributing to the SGS.

\section{Discussion}

The fine-scale SGS of P. cuneifolia was substantially strengthened by phenological barriers to pollenmediated gene flow along a snowmelt gradient. The $S p$ value among co-flowering pairs ( $S p=0.012 \pm 0.005$ s.e.) is similar to the value observed in other self-incompatible species $(S p=0.013$ across 17 species; Vekemans and Hardy, 2004). In contrast, the Sp value among non-coflowering pairs $(0.055 \pm 0.014)$ was about four times larger than the average value across species, indicating a steep decrease in the kinship coefficients with spatial distance. Assuming ideal populations have same $S p$ value for observed correlograms, Wright's neighborhood sizes as reciprocal of the $S p$ values (Vekemans and Hardy, 2004) increased with the extent of flowering overlap $\left(N_{\mathrm{b}}=10,45\right.$ and 81 for non-co-flowering pairs, control pairs and co-flowering pairs, respectively). Because the neighborhood size is originally defined as $4 \pi D \sigma^{2}$, where $D$ is population density and $\sigma^{2}$ is second moment of the distance between parents and offspring (Wright, 1943, 1946), larger neighborhood size partitioned by co-flowering pairs should result from increase in pollen-mediated gene flow if population density is constant. However, effect of phenological segregation on 
a
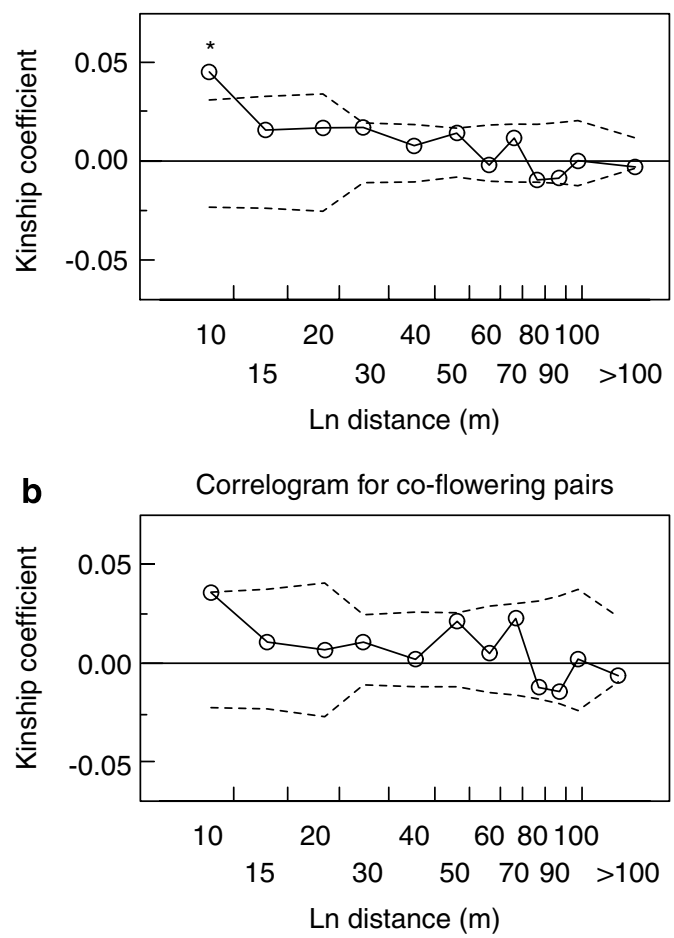

C

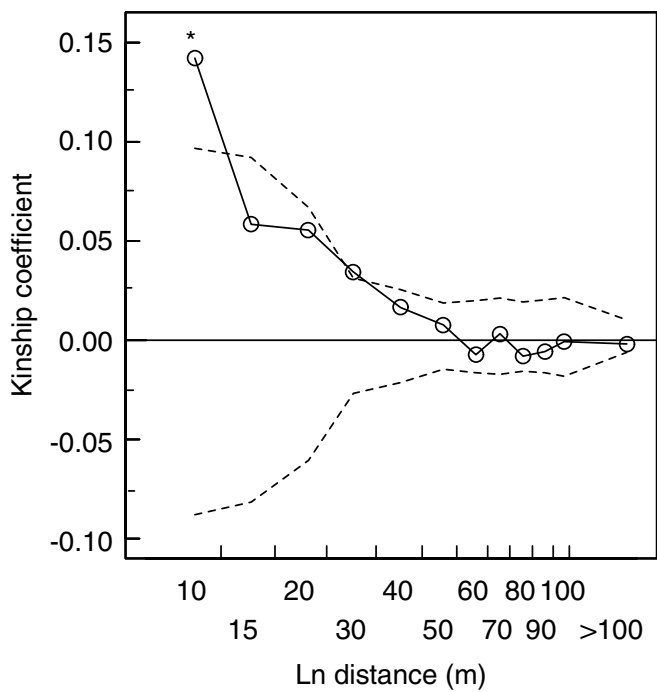

Figure 3 Correlogram of kinship coefficient among pairs of Primula cuneifolia individuals, as a function of pairwise distance class on a logarithmic scale. (a) Correlogram of all pairwise individuals (upper limit of the distance class: 10, 15, 20, 30, 40, 50, 60, 70, 80, 90,100 and $>100 \mathrm{~m}$ ), respectively. (b) Correlogram of pairwise individuals with co-flowering time ( $\leqslant 10$ days). (c) Correlogram of pairwise individuals with non-co-flowering time ( $>10$ days). Dashed lines represent a 95\% null hypothesis confidence region assuming no genetic structure based on 1000 randomizations. Asterisks indicate significance at the 5\% probability level.

the SGS was not detected by the partial Mantel test. This may be because of strong correlation between flowering phenology and geographical feature in our system (Figure 2a and $b$ ).

Probability of pollen-mediated gene flow may be influenced by the extent of flowering overlap among plants. If so, the SGS may break down when flowering
Table 2 Effect of flowering segregation on $S p$ statistics in Primula cuneifolia

\begin{tabular}{lccc}
\hline Pairwise individuals & $F$ & $b$ & $S p$ \\
\hline Control pairs & $0.045^{*}$ & $-0.016^{* *}$ & 0.017 \\
& $(0.010)$ & $(0.006$ & $(0.006)$ \\
& & & \\
Co-flowering pairs & 0.036 & $-0.012^{*}$ & 0.012 \\
& $(0.006)$ & $(0.006)$ & $(0.005)$ \\
& & & \\
Non-co-flowering pairs & $0.142^{* *}$ & $-0.047^{* *}$ & 0.055 \\
& $(0.019)$ & $(0.012)$ & $(0.014)$ \\
\hline
\end{tabular}

$F$, the mean kinship coefficient for the first distance class $(\leqslant 10 \mathrm{~m})$; $b$, the regression slope of kinship coefficients on the logarithmic physical distance interval.

The $S p$ statistic was computed as $-b /(1-F)$. The standard errors in parenthesis were calculated using a jackknife procedure over loci. Significance level: ${ }^{*} P<0.05 ;{ }^{* *} P<0.001$.

synchrony is high among plants, resulting in low $S p$ value. In this study, we categorized co-flowering based on standard flowering period of individual plants (that is, $\leqslant 10$ days). Even when tighter phenological criteria ( 3 or 5 days) were used for the analyses of SGS, however, results of $S p$ statistics and partial Mantel test were not influenced (data not shown). Because the progress of flowering sequence over the grid varies from year to year depending on the pace of snowmelt, the intensity of flowering overlap among plants should vary to some extent. Therefore, our phenological criterion based on standard flowering period (10 days) seems to be valid.

In our snowbed system, phenological barriers to gene flow is effective for pollen dispersal but not for seed dispersal. In previous work using a similar system (Stanton et al., 1997), the effect of flowering phenology on the genetic structure in Ranunculus adoneus was not clear, probably due to effective seed migration. In contrast with $R$. adoneus, evidence of segregation in flowering time of $P$. cuneifolia may imply limited effectiveness of seed dispersal. Although the distances that pollen and seed of $P$. cuneifolia are dispersed are unknown, the contemporary gene flow of another Primula species, P. sieboldii, has been reported (Ishihama et al., 2003; Washitani et al., 2005); mean and maximum distances for pollen dispersal from an experimental population were 7.2 and $89 \mathrm{~m}$, respectively, and seeds were dispersed c. $10 \mathrm{~cm}$ from the maternal plants. The range of the effective neighborhood estimated in $P$. sieboldii largely corresponds to the fine-scale genetic structure of $P$. cuneifolia, in which significant positive autocorrelations occurred within $10 \mathrm{~m}$. Efficient gene flow through the pollination process should reflect the foraging range and pollination efficiency of bumblebees. On the other hand, highly localized seed dispersal is a substantial force to generate a concentration of relatives (for example, Epperson and Alvarez-Buylla, 1997), which promotes frequent biparental inbreeding that contributes to mating among relatives in the overall level of inbreeding. In strictly outcrossing species, the kinship coefficient among adjacent individuals, $F$, can be compared to a maximum estimate for biparental inbreeding (Vekemans and Hardy, 2004). The high $F$ values among non-co-flowering pairs $(0.142 \pm 0.019)$ in comparison with that among co-flowering pairs $(0.036 \pm 0.009)$ should reflect that highly restricted pollen dispersal caused by phenological segregation, in 
addition to localized seed dispersal, accelerates the high degree of kinship structure in the first distance class. A simulation study predicted that highly restricted pollen dispersal can result in a high level of spatial autocorrelation (that is, the degree of genetic relatedness), especially around a neighborhood (Ohsawa et al., 1993). Our result supports this prediction.

Biparental inbreeding and local genetic structure can affect the range of effective gene dispersal and offspring fitness (Ishihama et al., 2005). In this obligate outcrossing species, identity by descent within individuals (that is, inbreeding coefficient) results from only biparental inbreeding. The observed inbreeding coefficient across all individuals $(F=0.063 \pm 0.027$ s.e.; Table 1$)$ was lower than the approximation of biparental inbreeding among non-co-flowering pairs $(F=0.142 \pm 0.019$ s.e.). This large reduction in the inbreeding component from the mating stage to the adult stage $(0.142-0.063=0.079)$ suggests that inbreeding depression could occur when spatially restricted pollen flow accelerates the mating events between related individuals (Ritland, 1990; Vekemans and Hardy, 2004). Note, however, that the low level of genetic polymorphism in this study limited our accurately estimating biparental inbreeding. If heterogeneity in flowering time increased the mean level of inbreeding depression at the population level, natural selection might drive to accelerate flowering overlap among individuals. Although spatial pattern of snowmelt is relatively consistent, the rate of snowmelt progress highly fluctuates from year to year in this area (Kudo and Hirao, 2005). Therefore, the environmental fluctuation may overwhelm the selective force for flowering phenology.

Spatial and temporal patterns of gene flow with respect to landscape features have crucial effects on the spatial patterns of genetic variation (Sork et al., 1999; Manel et al., 2003; Storfer et al., 2007). In an alpine region, the snowmelt gradient is a critical landscape feature affecting genetic structure of plants (Hirao and Kudo, 2004). Our study demonstrates that flowering segregation among individual plants substantially reinforces the SGS even in a continuous snow patch. Although contemporary pollen dispersal often varies depending on ecological factors such as population density (Levin and Kerster 1969), flowering phenology (Schmitt, 1983; Kitamoto et al., 2006) and seasonal changes in pollinator activity (Hirao et al., 2006), the historical gene flow across generations appears to be consistent in shaping the finescale SGS along a snowmelt gradient. Furthermore, the larger scale analyses of genetic structure in several alpine herb species (Peucedanum multivittatum, Veronica stelleri and Gentiana nipponica (Hirao and Kudo, 2004); Erythronium grandiflorum (Yamagishi et al., 2005)) revealed that the effect of snowmelt gradient on genetic structures varied among species. Overall genetic processes including the breeding system, population dynamics, genetic drift and selective force must be considered to understand hierarchical genetic structures and their significance for evolutionary potential.

\section{Acknowledgements}

We thank T Kasagi for support during the field survey and Y Kameyama for comments on the paper. This study was partly supported by a grant-in-aid from the Japanese Ministry of Education, Culture, Sports, Science, and
Technology for the 21st Century of Excellence Program (E-01), a grant-in-aid from the Ministry of Environment for the Global Environment Research Fund (F-052) and the Japan Society for the Promotion of Science (no. 16370007).

\section{References}

Degen BE, Bandou E, Caron H (2004). Limited pollen dispersal and biparental inbreeding in Symphonia globulifera in French Guiana. Heredity 93: 585-591.

Dutech C, Sork VL, Irwin AJ, Smouse PE, Davis FW (2005). Gene flow and fine-scale genetic structure in a wind-pollinated tree species, Quercus lobata (Fagaceae). Am J Bot 92: 252-261.

Epperson BK (1993). Recent advances in correlation analysis of spatial patterns of genetic variation. Evol Biol 27: 95-155.

Epperson BK, Alvarez-Buylla ER (1997). Limited seed dispersal and genetic structure in life stages of Cecropia obtusifolia. Evolution 51: 275-282.

Grifin CAM, Eckert CG (2003). Experimental analysis of biparental inbreeding in a self-fertilizing plant. Evolution 57: 1513-1519.

Goudet J (1995). FSTAT (version 1.2): a computer program to calculate F-statistics. J Heredity 86: 485-486.

Hamrick JL, Murawski DA, Nason JD (1993). The influence of seed dispersal mechanisms on the genetic structure of tropical tree populations. Vegetatio 107/108: 281-297.

Hardy OJ, Maggia L, Bandou E, Breyne P, Caron H, Chevallier $\mathrm{M}-\mathrm{H}$ et al. (2006). Fine-scale genetic structure and gene dispersal inferences in 10 neotropical tree species. Mol Ecol 15: 559-572.

Herlihy CR, Eckert CG (2004). Experimental dissection of inbreeding and its adaptive significance in a flowering plant, Aquilegia canadensis (Ranunculaceae). Evolution 58: 2693-2703.

Heywood JS (1991). Spatial analysis of genetic variation in plant populations. Annu Rev of Ecol Syst 22: 335-355.

Hirao AS, Kameyama Y, Ohara M, Isagi Y, Kudo G (2006). Seasonal changes in pollinator activity influence pollen dispersal and seed production of the alpine shrub Rhododendron aureum (Ericaceae). Mol Ecol 15: 1165-1173.

Hirao AS, Kudo G (2004). Landscape genetics of alpine snowbed-plants: comparisons along geographic and snowmelt gradients. Heredity 93: 290-298.

Holway JG, Ward RT (1965). Phenology of alpine plants in northern Colorado. Ecology 46: 73-83.

Ishihama F, Nakano C, Ueno S, Ajima M, Tsumura Y, Washitani I (2003). Seed set and gene flow patterns in an experimental population of an endangered heterostylous herb with controlled local opposite-morph density. Func Ecol 17: 680-689.

Ishihama F, Ueno S, Tsumura Y, Washitani I (2005). Gene flow and inbreeding depression inferred from fine-scale genetic structure in an endangered heterostylous perennial, Primula sieboldii. Mol Ecol 17: 680-689.

Kitamoto N, Ueno S, Takenaka A, Tsumura Y, Washitani I, Ohsawa R (2006). Effect of flowering phenology on pollen flow distance and the consequences for spatial genetic structure within a population of Primula sieboldii (Primulaceae). Am J Bot 93: 226-233.

Kudo G (1991). Effects of snow-free period on the phenology of alpine plants inhabiting snow patches. Arc Alp Res 23. 436-443.

Kudo G (1992). Pre-flowering and fruiting periods of alpine plants inhabiting a snow-bed. J Phytogeogr Taxon 40: 99-106.

Kudo G (1996). Effects of snowmelt timing on reproductive phenology and pollination process of alpine plants. Memory of National Institute of Polar Research (Tokyo) Special Issue 51 $71-82$.

Kudo G, Hirao AS (2005). Habitat-specific responses in the flowering phenology and seed set of alpine plants to climate 
variation: implications for global-change impacts. Population Ecology 48: 49-58.

Levin DA, Kerster HW (1969). The dependence of bee-mediated pollen and gene dispersal upon plant density. Evolution 23: 560-571.

Loiselle BA, Sork VL, Nason J, Graham C (1995). Spatial genetic structure of tropical understory shrub, Psychotria officinalis (Rubiaceae). Am J Bot 82: 1420-1425.

Manel S, Schwartz MK, Luikart C, Taberlet P (2003). Landscape genetics: combining landscape ecology and population genetics. Trends Ecol Evol 18: 189-197.

Moran PAP (1950). Notes on continuous stochastic phenomena. Biometrika 37: 17-23.

Ohsawa R, Furuya N, Ukai Y (1993). Effect of spatially restricted pollen flow on spatial genetic structure of an animalpollinated allogamous plant population. Heredity 71: 64-73.

$\mathrm{R}$ Development Core Team (2006). R: A Language and Environment for Statistical Computing. R Foundation for Statistical Computing: Vienna, Austria http:/ / www.R-project.org.

Richards AJ (2002). Primula 2nd edn. BT Batsford: London, UK.

Ritland K (1990). Inferences about inbreeding depression based on changes of the inbreeding coefficient. Evolution 44: 1230-1241.

Schmitt J (1983). Density-dependent pollinator foraging, flowering phenology, and temporal pollen dispersal patterns in Linanthus bicolor. Evolution 37: 1247-1257.

Shiraishi S (1988). Inheritance of isozyme variations in Japanese black pine, Pinus thunbergii Parl. Silvae Genet 37: 93-100.

Sork VL, Nason J, Campbell DR, Fernandez JF (1999). Landscape approaches to historical and contemporary gene flow in plants. Trends Ecol Evol 14: 219-224.
Stanton ML, Galen C, Shore J (1997). Population structure along a steep environmental gradient: consequences of flowering time and habitat variation in the snow buttercup, Ranunculus adoneus. Evolution 51: 79-94.

Storfer A, Murphy MA, Evans JS, Goldberg CS, Robinson S, Spear SF et al. (2007). Putting the 'landscape' in landscape genetics. Heredity 98: 128-142.

Tsumura Y (2001). Allozyme experiment method (in Japanese).In: The Society for the Study of Species Biology (eds). Molecular Ecology of Woody Species. Bun-ichi Sogo Shyuppan: Tokyo, Japan, pp 183-219.

Turner ME, Claiborne JC, Anderson WW (1982). Homozygosity and patch structure in plant populations as a result of nearest-neighbor pollination. Proc Natl Acad Sci USA 79: 203-207.

Vekemans X, Hardy OJ (2004). New insights from fine-scale spatial genetic structure analyses in plant populations. Mol Ecol 13: 921-935.

Washitani I, Ishihama F, Shimono A, Nishihiro MA (2005). Toward predicting gene flow in plant populations. Plant Biotechnol 22: 489-495.

Weir BS, Cokerham CC (1984). Estimating F-statistics for the analysis of population structure. Evolution 38: $1358-1370$.

Wright S (1943). Isolation by distance. Genetics 28: 114-138.

Wright S (1946). Isolation by distance under diverse systems of mating. Genetics 31: 39-59.

Yamagishi H, Ohara M, Allison TD (2005). Effect of snowmelt timing on the genetic structure of an Erythronium grandiflorum population in an alpine environment. Ecol Res 20: 199-204. 